\title{
BEST UNIFORM APPROXIMATION BY BOUNDED ANALYTIC FUNCTIONS
}

\author{
M. PAPADIMITRAKIS
}

(Communicated by Irwin Kra)

\begin{abstract}
This paper gives a counterexample to the conjecture that the continuity of the conjugate $\tilde{f}$ of an $f \in C(T)$ implies the continuity of the best uniform approximation $g \in H^{\infty}(T)$ of $f$. It also states two conditions which imply the continuity of $g$.
\end{abstract}

Let $L^{\infty}(T)$ the space of bounded measurable functions on the unit circle $T$, $H^{\infty}(T)$ the subalgebra of $L^{\infty}(T)$ consisting of nontangential limits of bounded analytic functions in the unit disk and write $\|f\|_{\infty}$ for the (essential supremum) norm of $f \in L^{\infty}(T)$. Also, let $C(T)$ be the space of all continuous functions on $T$.

It is known that any $f \in L^{\infty}(T)$ has at least one best approximation $g \in H^{\infty}(T)$, in the sense that

$$
d=\|f-g\|_{\infty}=\inf _{h \in H^{\infty}}\|f-h\|_{\infty}
$$

and that, by duality

$$
d=\sup \left\{\left|\int_{0}^{2 \pi} f(\theta) F(\theta) \frac{d \theta}{2 \pi}\right|: F \in H^{1}(T), F(0)=0,\|F\|_{1} \leq 1\right\}
$$

where $H^{p}(T)(0<p<\infty)$ is the Hardy space of all nontangential limits of functions $F$ analytic in the unit disc such that

$$
\|F\|_{p}^{p}=\sup _{0<r<1} \int_{0}^{2 \pi}\left|F\left(r e^{i \theta}\right)\right| \frac{d \theta}{2 \pi}<+\infty .
$$

Moreover, if $f$ is continuous, then the best approximation $g$ of $f$ is unique and there is at least one $F$, for which the supremum (*) is attained. Also $f, g$ and any of those maximizing $F$ 's are connected by

$$
f(\theta)-g(\theta)=\|f-g\|_{\infty} \frac{\overline{F(\theta)}}{|F(\theta)|} \text { a.e. }(d \theta)
$$

which implies

$$
|f(\theta)-g(\theta)|=\|f-g\|_{\infty}=d \quad \text { a.e. }(d \theta) .
$$

We need the following result (see [1 or 2$]$ ):

THEOREM 1 (CARLESON-JACOBS). If $f \in C(T), g \in H^{\infty}(T), F \in H^{1}(T)$ are connected by $(1)$, then

(a) $F \in H^{p}(T)$, for all $p<+\infty$,

Received by the editors May 19, 1987.

1980 Mathematics Subject Classification (1985 Revision). Primary 30D55; Secondary 42A50.

Partially supported by NSF Grant \# DMS85-03780.

(C)1988 American Mathematical Society $0002-9939 / 88 \$ 1.00+\$ .25$ per page 
(b) if $\tau \in[0,2 \pi]$ and if

$$
f_{\tau}(\theta)=f(\theta)-f(\tau), \quad g_{\tau}(\theta)=g(\theta)-f(\tau)
$$

then there is $\delta>0$ and $r_{0}>0$ such that

$$
\left|g_{\tau}(z)\right| \geq \frac{1}{2} \cdot\|f-g\|_{\infty} \quad \text { on } W_{\tau}=\left\{z=r e^{i \theta}:|\theta-\tau|<\delta, r_{0}<r<1\right\}
$$

where $\delta$ and $r_{0}$ can be independent of $\tau$.

We consider the problem of how the regularity of $f$ affects the regularity of $g$. In [1] the following is proved.

THEOREM 2. If $f$ is Dini-continuous, i.e. if $\int_{0}(\omega(t) / t) d t<+\infty$, where $\omega(t)=$ $\sup _{|x-y| \leq t}|f(x)-f(y)|$ is the modulus of continuity of $f$, then its best approximation $g$ is also continuous.

In [1] a function $f$ is constructed, continuous but not Dini-continuous, whose best approximation $g$ is not continuous.

Because the Dini-continuity of $f$ implies the continuity of its conjugate $\tilde{f}$ and because of the proof in [1], it was conjectured that, for $f \in C(T)$, the continuity of $\tilde{f}$ and the continuity of $g$ are equivalent.

It was proved by Sarason that the continuity of $g$ does not imply the continuity of $\tilde{f}$. See $[2$, p. 177].

This paper provides a counterexample for the other half of the conjecture. It constructs a continuous function $f$, whose conjugate $\tilde{f}$ is continuous, but whose best approximation $g$ is not. We also give two further conditions on $f$ which imply $g$ is continuous.

In the following $\bar{f}$ is the complex conjugate of $f$.

THEOREM 3. If $\bar{f} \in A(T)=H^{\infty}(T) \cap C(T)$ and $\int_{0}\left(\omega^{2}(t) / t\right) d t<+\infty$, then $g$, the best approximation of $f$, is continuous.

THEOREM 4. If $\bar{f} \in A(T)$ and $\widetilde{|f|^{2}} \in C(T)$ and $\int_{0}\left(\omega^{3}(t) / t\right) d t<+\infty$, then $g$ is continuous.

THEOREM 5. There exists a function $f$, such that $\bar{f} \in A(T)$, but such that its best approximation $g$ is not continuous.

Since $\tilde{f}=-i f$ when $\bar{f} \in A(T)$, the function in Theorem 5 has a continuous conjugate.

ProOF OF THEOREM 3. Suppose $\|f-g\|_{\infty}=1$. Fix $\tau \in[0,2 \pi]$. Then, from Theorem 1(b), $g_{\tau}(z)$ has a well-defined logarithm on $W_{\tau}$, which is given by

$$
\log g_{\tau}(z)=\frac{1}{2 \pi} \int_{|\theta-\tau| \leq \delta} \log \left|g_{\tau}(\theta)\right| \cdot \frac{e^{i \theta}+z}{e^{i \theta}-z} d \theta+R_{\tau}(z), \quad z \in W_{\tau}
$$

where $R_{\tau}(z)$ is the integral over $|\theta-\tau|>\delta$ plus the logarithm of the inner factor of $g_{\tau}$. Since $\left|g_{\tau}\right| \geq \frac{1}{2}$ on $W_{\tau}$, this inner factor is analytic across $|\theta-\tau|<\delta$. So $R_{\tau}(z)$ and its derivative are bounded on $\left|z-e^{i \tau}\right|<\delta_{1}$, for some $\delta_{1}<\delta$, independent of $\tau$. This implies

$$
\left|R_{\tau}(z)-R_{\tau}(w)\right| \leq c|z-w| \text { for }\left|z-e^{i \tau}\right|<\delta_{1},\left|w-e^{i \tau}\right|<\delta_{1} .
$$


We also have

$$
|f(\theta)-g(\theta)|=1 \quad \text { a.e. }(d \theta)
$$

from which

$$
\left|f_{\tau}(\theta)-g_{\tau}(\theta)\right|=1 \quad \text { a.e. }(d \theta)
$$

and

$$
\left|g_{\tau}\right|^{2}=1+2 \cdot \operatorname{Re}\left(\bar{f}_{\tau} \cdot g_{\tau}\right)-\left|f_{\tau}\right|^{2}
$$

Therefore

$$
\begin{aligned}
\log \left|g_{\tau}\right| & =\frac{1}{2} \log \left|g_{\tau}\right|^{2}=\frac{1}{2}\left[2 \cdot \operatorname{Re}\left(\bar{f}_{\tau} \cdot g_{\tau}\right)-\left|f_{\tau}\right|^{2}+O\left(\left|f_{\tau}\right|^{2}\right)\right] \\
& =\operatorname{Re}\left(\bar{f}_{\tau} g_{\tau}\right)+O\left(\left|f_{\tau}\right|^{2}\right)
\end{aligned}
$$

and

$$
\begin{aligned}
\log g_{\tau}(z)= & \frac{1}{2 \pi} \int_{|\theta-\tau| \leq \delta} \operatorname{Re}\left(\bar{f}_{\tau} g_{\tau}\right) \frac{e^{i \theta}+z}{e^{i \theta}-z} d \theta \\
& +\frac{1}{2 \pi} \int_{|\theta-\tau| \leq \delta} O\left(\left|f_{\tau}\right|^{2}\right) \frac{e^{i \theta}+z}{e^{i \theta}-z} d \theta+R_{\tau}(z) .
\end{aligned}
$$

Since $\bar{f}_{\tau}$ is analytic, $\bar{f}_{\tau} g_{\tau}$ is also analytic, which implies that

$$
\frac{1}{2 \pi} \int_{0}^{2 \pi} \operatorname{Re}\left(\bar{f}_{\tau} g_{\tau}\right) \frac{e^{i \theta}+z}{e^{i \theta}-z} d \theta=\bar{f}_{\tau}(z) \cdot g_{\tau}(z) \text {. }
$$

Thus:

$$
\log g_{\tau}(z)-\bar{f}_{\tau}(z) g_{\tau}(z)=\frac{1}{2 \pi} \int_{|\theta-\tau| \leq \delta} O\left(\left|f_{\tau}\right|^{2}\right) \frac{e^{i \theta}+z}{e^{i \theta}-z} d \theta+R_{\tau}^{*}(z)
$$

where

$$
R_{\tau}^{*}(z)=R_{\tau}(z)-\frac{1}{2 \pi} \int_{|\theta-\tau|>\delta} \operatorname{Re}\left(\bar{f}_{\tau} g_{\tau}\right) \frac{e^{i \theta}+z}{e^{i \theta}-z} d \theta
$$

and so, by (2),

$$
\left|R_{\tau}^{*}(z)-R_{\tau}^{*}(w)\right| \leq c|z-w| \text { for }\left|z-e^{i \tau}\right|<\delta_{1},\left|w-e^{i \tau}\right|<\delta_{1} .
$$

If $z$ is in a truncated cone $\Gamma(\tau)$, which is inside $\left|z-e^{i \tau}\right|<\delta_{1}$ and has vertex $e^{i \tau}$, then

$$
\left|\frac{e^{i \theta}+z}{e^{i \theta}-z}\right|<\frac{c}{|\theta-\tau|}
$$

and so

$$
\left|\log g_{\tau}(z)-\bar{f}_{\tau}(z) g_{\tau}(z)-R_{\tau}^{*}(z)\right| \leq c \cdot \int_{0}^{\delta} \frac{\omega^{2}(t)}{t} d t .
$$

Since $\bar{f}_{\tau}(z) \rightarrow 0$ as $z \rightarrow e^{i \tau}$,

$$
\left|\log g_{\tau}(z)-R_{\tau}^{*}(z)\right| \leq c \int_{0}^{\delta} \frac{\omega^{2}(t)}{t} d t+\eta(\delta)
$$

where $\eta(\delta) \rightarrow 0$ as $\delta \rightarrow 0$. Hence, by (3),

$$
\left|g_{\tau}(z)-g_{\tau}(w)\right| \leq c|z-w|+\eta_{1}(\delta), \quad z, w \in \Gamma(\tau),
$$

where $\eta_{1}(\delta) \rightarrow 0$ as $\delta \rightarrow 0$. 
Now, if $\sigma$ and $\tau$ are close to each other and $z \in \Gamma(\tau) \cap \Gamma(\sigma)$ then

$$
\begin{aligned}
\left|g\left(e^{i \tau}\right)-g\left(e^{i \theta}\right)\right| & \leq\left|g\left(e^{i \tau}\right)-g(z)\right|+\left|g(z)-g\left(e^{i \sigma}\right)\right| \\
& =\left|g_{\tau}\left(e^{i \tau}\right)-g_{\tau}(z)\right|+\left|g_{\sigma}(z)-g_{\sigma}\left(e^{i \sigma}\right)\right| \\
& \leq c\left|e^{i \tau}-z\right|+c\left|e^{i \sigma}-z\right|+2 \eta_{1}(\delta) \leq c|\tau-\sigma|+2 \eta_{1}(\delta),
\end{aligned}
$$

and

$$
\varlimsup_{\sigma \rightarrow \tau}\left|g\left(e^{i \tau}\right)-g\left(e^{i \sigma}\right)\right| \leq 2 \eta_{1}(\delta)
$$

so that

$$
\lim _{\sigma \rightarrow \tau} g\left(e^{i \sigma}\right)=g\left(e^{i \tau}\right)
$$

and $g$ is continuous.

PROOF OF THEOREM 4. Now we carry the expansion of $\log \left|g_{\tau}\right|$ one step further:

$$
\begin{aligned}
\log \left|g_{\tau}\right|= & \frac{1}{2}\left[2 \operatorname{Re}\left(\bar{f}_{\tau} g_{\tau}\right)-\left|f_{\tau}\right|^{2}-\frac{\left(2 \operatorname{Re}\left(\bar{f}_{\tau} g_{\tau}\right)-\left|f_{\tau}\right|^{2}\right)^{2}}{2}+O\left(\left|f_{\tau}\right|^{3}\right)\right] \\
= & \operatorname{Re}\left(\bar{f}_{\tau} g_{\tau}\right)-\frac{1}{2}\left|f_{\tau}\right|^{2}-\left(\operatorname{Re}\left(\bar{f}_{\tau} g_{\tau}\right)\right)^{2}+O\left(\left|f_{\tau}\right|^{3}\right) \\
= & \operatorname{Re}\left(\bar{f}_{\tau} g_{\tau}\right)-\frac{1}{2}\left|f_{\tau}\right|^{2}-\frac{1}{2}\left|\bar{f}_{\tau} g_{\tau}\right|^{2}-\frac{1}{2} \operatorname{Re}\left(\bar{f}_{\tau} g_{\tau}\right)^{2}+O\left(\left|f_{\tau}\right|^{3}\right) \\
= & \operatorname{Re}\left(\bar{f}_{\tau} g_{\tau}\right)-\frac{1}{2} \operatorname{Re}\left(\bar{f}_{\tau} g_{\tau}\right)^{2}-\frac{1}{2}\left|f_{\tau}\right|^{2} \\
& -\frac{1}{2}\left|f_{\tau}\right|^{2}\left(1+2 \operatorname{Re}\left(\bar{f}_{\tau} g_{\tau}\right)-\left|f_{\tau}\right|^{2}\right)+O\left(\left|f_{\tau}\right|^{3}\right) \\
= & \operatorname{Re}\left(\bar{f}_{\tau} g_{\tau}\right)-\frac{1}{2} \operatorname{Re}\left(\bar{f}_{\tau} g_{\tau}\right)^{2}-\left|f_{\tau}\right|^{2}+O\left(\left|f_{\tau}\right|^{3}\right) .
\end{aligned}
$$

Now, because

$$
\frac{1}{2 \pi} \int_{0}^{2 \pi} \operatorname{Re}\left(\bar{f}_{\tau} g_{\tau}\right)^{2} \frac{e^{i \theta}+z}{e^{i \theta}-z} d \theta=\left(\bar{f}_{\tau}(z) g_{\tau}(z)\right)^{2}
$$

since $\bar{f} g \in H^{\infty}(T)$, we get

$$
\begin{aligned}
& \log g_{\tau}(z)-\bar{f}_{\tau}(z) g_{\tau}(z)+\frac{1}{2}\left(\bar{f}_{\tau}(z) g_{\tau}(z)\right)^{2} \\
& \quad=-\frac{1}{2 \pi} \int_{|\theta-\tau| \leq \delta}\left|f_{\tau}(\theta)\right|^{2} \frac{e^{i \theta}+z}{e^{i \theta}-z} d \theta+\frac{1}{2 \pi} \int_{|\theta-\tau| \leq \delta} O\left(\left|f_{\tau}\right|^{3}\right) \frac{e^{i \theta}+z}{e^{i \theta}-z} d \theta+R_{\tau}^{* *}(z)
\end{aligned}
$$

where

$$
R_{\tau}^{* *}(z)=R_{\tau}^{*}(z)+\frac{1}{2 \pi} \int_{|\theta-\tau|>\delta} \operatorname{Re}\left(\bar{f}_{\tau} g_{\tau}\right)^{2} \frac{e^{i \theta}+z}{e^{i \theta}-z} d \theta
$$

and so

$$
\left|R_{\tau}^{* *}(z)-R_{\tau}^{* *}(w)\right| \leq c|z-w| \text { for }\left|z-e^{i \tau}\right| \leq \delta_{1},\left|w-e^{i \tau}\right| \leq \delta_{1} .
$$

Now, the continuity of $\widetilde{|f|^{2}}$ implies the continuity of $\mid \widetilde{\left.f_{\tau}\right|^{2}}$, and this implies the continuity of the first integral. The rest of the proof proceeds as in Theorem 3.

PROOF OF THEOREM 5. Consider the function

$$
\begin{aligned}
u(t) & =-\alpha_{1} \log |\log t|, & 0<t<\frac{1}{2}, \\
& =-\alpha_{2} \log |\log | t||, \quad & -\frac{1}{2}<t<0,
\end{aligned}
$$

extended to be smooth in $[-\pi, \pi]-\{0\}$, and consider the harmonic extension $u(z)$ of $u(t)$ inside the unit disk, its conjugate $\tilde{u}(z)$ and $f(z)=e^{u(z)-i \tilde{u}(z)}$. 
Then, since $\tilde{u}(t)$ is continuous in $[-\pi, \pi]-\{0\}$, and $|f(z)|=e^{u(z)} \rightarrow 0$ as $z \rightarrow 1$, we see that $\bar{f} \in A(T)$.

If $\frac{1}{3}<\alpha_{1} \leq \frac{1}{2}$ and $\frac{1}{2}<\alpha_{2}$, then

$$
\int_{0}^{1 / 2} \frac{|f(t)|^{3}}{t} d t<+\infty \text { and } \int_{-1 / 2}^{0} \frac{|f(t)|^{3}}{|t|} d t<+\infty
$$

but

$$
\int_{0}^{1 / 2} \frac{|f(t)|^{2}}{t} d t=+\infty \text { and } \int_{-1 / 2}^{0} \frac{|f(t)|^{2}}{|t|} d t<+\infty
$$

The last two imply that

$$
\widetilde{|f|^{2}}(r) \rightarrow+\infty \quad \text { as } r \rightarrow 1-
$$

From

$$
\begin{aligned}
& \log g(r)-\bar{f}(r) g(r)+\frac{1}{2}(\bar{f}(r) \cdot g(r))^{2} \\
& \quad=|f|^{2}(r)+i \widetilde{|f|^{2}}(r)+\frac{1}{2 \pi} \int_{|\theta-\tau| \leq \delta} O\left(|f|^{3}\right) \frac{e^{i \theta}+r}{e^{i \theta}-r} d \theta+R^{* *}(r)
\end{aligned}
$$

we get that

$$
\arg g(r) \rightarrow+\infty \quad \text { as } r \rightarrow 1-
$$

Thus $g$ is not continuous.

\section{REFERENCES}

1. L. Carleson and S. Jacobs, Best uniform approximation by analytic functions, Ark. Mat. 10 (1972), 219-229.

2. J. Garnett, Bounded analytic functions, Academic Press, New York, 1981.

Department of Mathematics, University of California, los Angeles, CaliFORNIA 90024

Current address: Department of Mathematics, University of Wisconsin-Madison, Madison, Wisconsin 53706 